\title{
Two-cell correlations in biological tissues
}

\author{
J. C. M. Mombach, R. M. C. de Almeida, and J. R. Iglesias \\ Instituto de Física, Universidade Federal do Rio Grande do Sul, Caixa Postal 15051, 91501-970 Porto Alegre, \\ Rio Grande do Sul, Brazil
}

(Received 8 December 1992)

\begin{abstract}
We present two-cell correlation functions $m_{l}(n)$, which give the average number of $l$-sided cells adjacent to $n$-sided ones, obtained experimentally from vegetable tissues and through a numerical simulation that includes mitosis of biological-tissue growth. The correlation functions are not always linear in $n$, but the Aboav-Weaire law is obeyed, indicating that it is valid for biological tissues and that recent arguments applied to purely topological models are not valid for all natural systems.
\end{abstract}

PACS number(s): 87.10.+e, 82.70.Rr

Soap bubbles, polycrystalline grains, and biological tissues present many similar features, in spite of arising from different natural phenomena. The common characteristics become apparent when they are mapped onto two-dimensional, space-filling, disordered cellular structures that are described through distribution functions (and their moments) that give, for example, the relative number of cells with a given number of sides $n$, area $a$ and perimeter $p$. This common behavior is expected because topology, geometry, and also growth dynamics impose some constraints that definitely influence the final pattern [1-3]: topology requires that every two-dimensional, space-filling cellular net of coordination number three on a flat surface presents an average number of sides $\langle n\rangle$ equal to six, geometry imposes a maximum possible area for a cell with given perimeter, and growth dynamics requires minimization of a cost function that can be either the grain boundary energy for polycrystalline grains, or the optimization of the (area)/(perimeter) ratio in the case of biological tissues [4]. While the growth dynamics of soap froths favors the formation of an inhomogeneous system with a few large many-sided cells embedded in a big number of small, 3-, 4-, or 5-sided cells, topology requires $\langle n\rangle=6$, hence favoring a relatively homogeneous system. On the other hand, the growth dynamics of vegetable tissues, where mitosis plays the main role, generates systems with low area dispersion. This interdependence of topology, geometry, and growth dynamics determines the final pattern of the cellular structure. Of course a very different growth dynamics may lead to other distributions functions, but similarities in natural systems indicates that the final structure is robust against minor changes in growth dynamics, that is, it seems that the minimization of total interface and a ban on collapsed (zero area) cells are sufficient conditions to reproduce the cellular patterns found in nature [3].

Some theoretical work has been done on the subject (see [3,5-7] and references therein); also, a striking empirical universality appears in numerical simulations [815] and in experimental results [16-19]: consider $m(n)$, the average number of sides of the neighbors of an $n$-sided cell. It is observed that

$$
n m(n)=(6-a) n+\left(6 a+\mu_{2}\right)
$$

where $a$ is always close to 1 in experiments, yielding a slope near 5 , and $\mu_{2}$ is the second moment of side distribution, with the average $\langle n\rangle=6$, imposed by topological constraints [2]. This relation is known as the AboavWeaire law and reveals a strong correlation between the number of sides of neighboring cells. Its origin is not completely understood; some authors have provided arguments for the form of this law based on geometry [2] or local screening of the topological charge [20].

In a recent paper, Peshkin, Strandburg, and Rivier [21] assert that the microreversibility argument for the universality, generally accepted as a theoretical support for the Aboav-Weaire law, may be incorrect. Their "disproof" considers two elementary topological transformations (three-sided cell disappearance and neighborswitching processes) and two-cell correlation functions $m_{l}(n)$, associated with the probability of finding an $l$ sided cell neighboring an $n$-sided one. They showed that only in the case of independent rates of each elementary topological transformation do the two-cell correlation functions yield a linear dependence of $n m(n)$ vs $n$, as stated by the Aboav-Weaire law, with slopes that vary from 5 to 7.12. But independent rates are highly improbable precisely because of the correlation between adjacent cells imposed by Eq. (1).

In biological tissues a third elementary topological transformation may also happen: mitosis. The origin of mitosis is a competition between the capacity of the cellular membrane to absorb nutrients $[\sim$ (perimeter)] and the required amount of these nutrients to keep the cell alive $[\sim$ (area) in two-dimensional tissues]. The mitosis process guarantees an optimal ratio of area to perimeter and implies a constant average cell area. Following the same reasoning as in Ref. [21] it is straightforward to show that the constraints required by the Aboav-Weaire law are even more severe when mitosis is taken into account: in this case three elementary topological transformations must have independent rates to yield a linear dependence of $n m(n)$ vs $n$.

Equation (1) can be calculated from the two-cell correlation functions $m_{l}(n)$ that give the average number of $l$-sided cells neighboring $n$-sided ones. These correlation functions have received increasing attention as a possible reason for the universality of the Aboav-Weaire law, and clearly experimental and numerical data are inter- 
esting. Here we present the two-cell correlation functions $m_{l}(n)$ for biological tissues obtained in experiments and through numerical simulations.

Experimental data have been obtained from vegetable leaves, following the procedures described in Ref. [19]. Vegetable tissues may present cellular structures with different patterns, signaling the effect of additional constraints, probably originated in the particular growth mechanism, as shown in Fig. 1: typical samples of the observed tissues from Ref. [19] may present either extended cells, differing from soap froths or metallurgical aggregates, or isotropic structures, indicating that the particular growth mechanism does not favor a given direction. The plot of $n m(n)$ vs $n$ for all five samples is shown in Fig. 2: it is evident that, in spite of the difference between the tissues, the Aboav-Weaire law is obeyed in all cases.

We also present, for the first time for biological tissues, the two-cell correlation functions $m_{l}(n)$. Figure 3 shows the plots of $m_{5}(n), m_{6}(n)$, and $m_{7}(n)$ for $A$. arborescens and $A$. attenuata; data regarding $m_{4}(n)$ and $m_{8}(n)$ were not statistically satisfactory and are not shown. In Fig. 4 we present the experimental data for $c f$. Anthurium, numerical-simulation results (both structures have similar $\left.\mu_{2}\right)$, and two-cell correlation functions for soap froths $\left(\mu_{2}=2.49\right)$, calculated from the experimental correlatedside distributions obtained by Glazier, Anderson, and Grest [8]: the three sets of data show similar behaviors. $A$. sativum and $A$. cepa [Figs. 1(a) and 1(b)], which were also investigated in Ref. [19], presented too few cells to allow any reliable measures of two-cell correlation functions. However, they showed the same tendency in spite of their anisotropic features. This anisotropy probably originated in additional biological mechanisms in the growth dynamics, i.e., some kind of global symmetry axis. In that sense biological tissues differ from soap froths where the network rapidly reorganizes to recreate isotropic bubbles when subjected to shear [22]. But two-cell correlations seem not to be affected when the two-dimensional space embedding the cellular structure is stretched in one given direction: the cells get longer in that direction but the number of neighbors of each cell remains the same. The anisotropy clearly affects distributions in area and perimeter [19]: the structure is more homogeneous and minimization of interfacial area is not a major factor to explain those patterns. On the other hand, isotropic samples are well described by a simulation based on surface-energy minimization that will be discussed in what follows.

Numerical simulations have been performed by considering a square lattice with a label being associated to each site. Zero sites are associated to empty space. Any connected set of sites with the same label is considered as one cell, the area being the number of sites of the set and perimeter being the number of edges separating two differently labeled sites. The simulation was performed in a Sun Sparcstation 2 and the initial state is a unique cell of about 100 sites, surrounded by empty space (zeros).

In each simulation step a site and a first neighbor are randomly chosen. When the neighbor is empty a new site and neighbor are chosen. When the neighbor is not empty (and the site may or not be empty) a local-energy difference $\Delta E$ is calculated, energy being proportional to the number of different neighbors surrounding a site (a zero-labeled neighbor implies zero energy). A local-energy difference determines the probability $(\min [1, \exp -\beta \Delta E])$ that the site label switches to the neighbor label and $\beta$ is an external noise parameter or temperature: $\beta=T^{-1}$. The present results were obtained with $T=0.4$. A Monte Carlo step is completed when the number of simulation steps is equal to the number of sites. During the growth process a cell may split into two or more pieces. After each Monte Carlo step a routine is run to locate all pieces of a given cell and attribute different labels. This procedure guarantees that cells are simply connected for all temperatures. Details

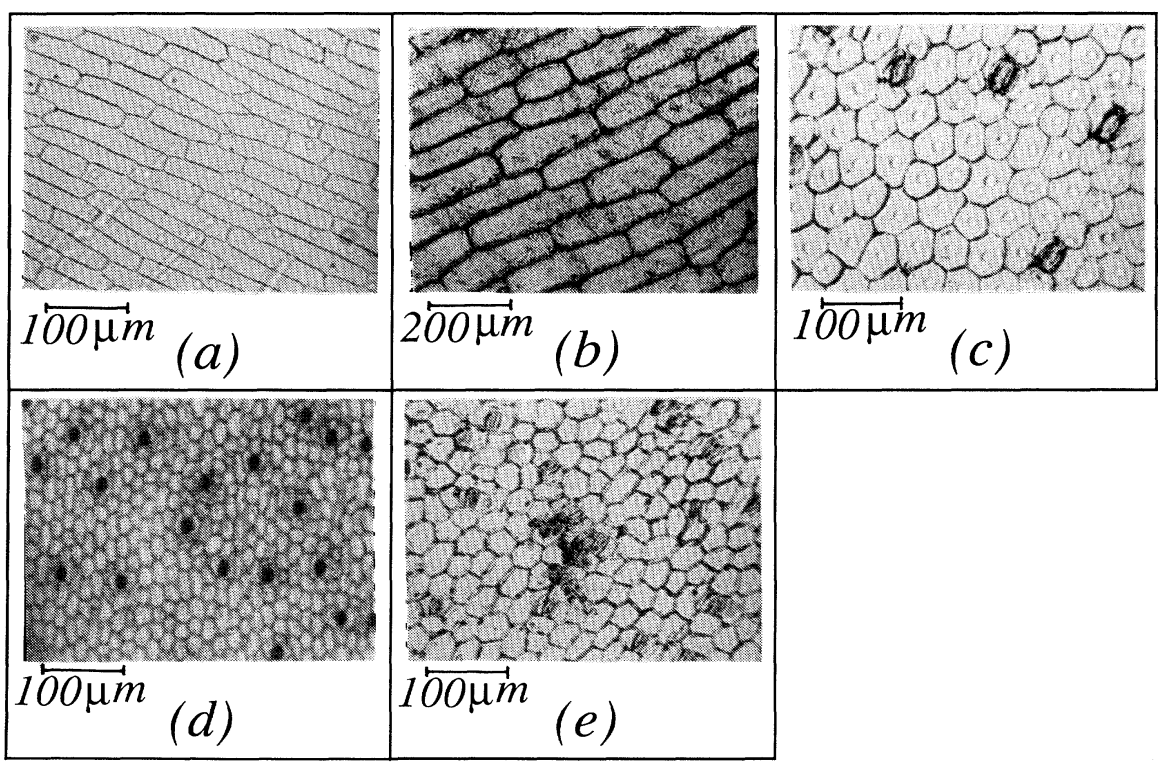

FIG. 1. Representative images of the epidermal tissue [19] for (a) $A$. sativum, (b) $A$. cepa, (c) A. attenuata, (d) $A$. arborescens, and (e) $c f$. Anthurium. 


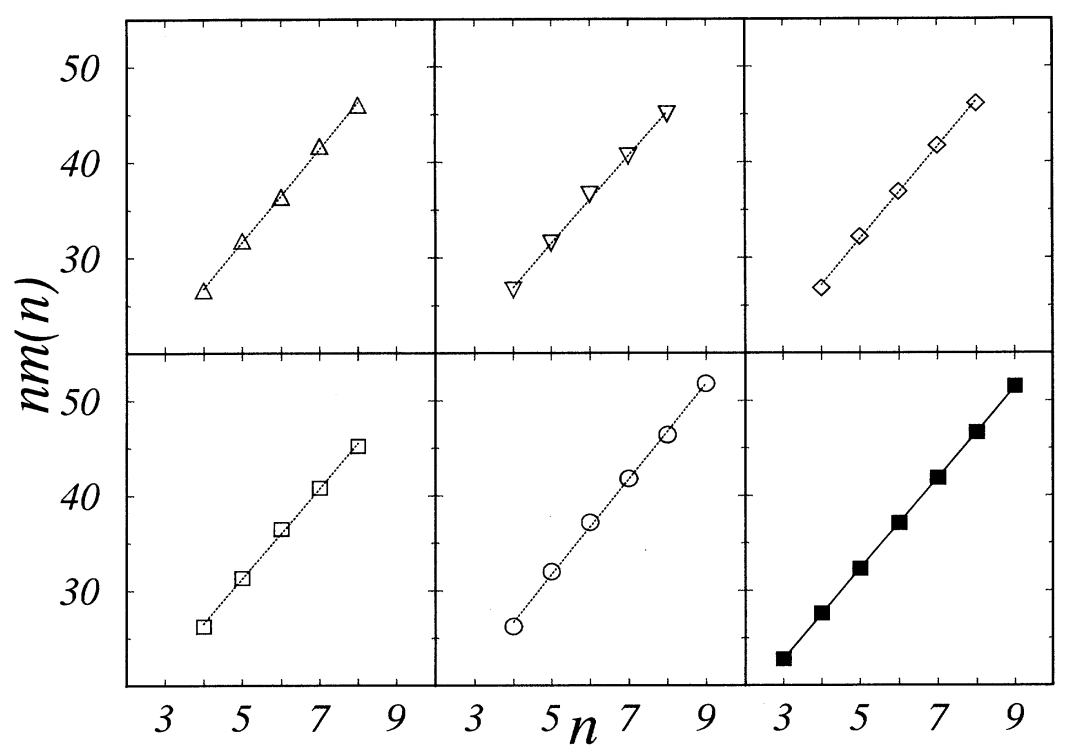

FIG. 2. Plot of $n m(n)$ as a function of $n$, obtained experimentally from $A$. sativum (triangles up), A. cepa (triangles down), $A$. attenuata (diamonds), $A$. arborescens (squares), cf. Anthurium (circles) samples, and from simulation (filled squares). The Aboav-Weaire distribution is verified with a slope of $4.9,4.6,4.8,4.8$, and 5 , respectively, for vegetable samples [19] and 4.8 for simulation results. about the simulation procedure and an extended discussion of the results will be published elsewhere [15].

A recent work by Graner and Glazier [23] also considers a Potts model to simulate biological-cell sorting; there a constant average cell area is obtained through an additional energy term that constrains the cell areas around a given value, but no cell division is performed. Here we explicitly consider cell division, which is triggered when the perimeter length is insufficient to supply the required amount of nutrients. After every Monte Carlo step a mitosis routine is run: the center of mass, area $a$, and perimeter $p$ of each cell are measured and whenever the ratio $a / p$ exceeds a given mitosis parameter $k$ (here $k=3$ ), the cell suffers mitosis, i.e., it is divided in two different cells by an edge crossing the center of mass in the direction of the least diameter, as occurs in real tissues [4]. This procedure has some similarities with the Potts-model simulation by Srolovitz and collaborators $[9,10]$, but it accounts for the differences between polycrystalline-grain and biological-tissue growth: a zero site may only become alive if it has a live neighbor and the cells may suffer mitosis. A stationary state is attained with invariant distribution functions and a constant average cell area.

Our interest here is two-cell correlations. The second moment of the tissue in this simulation is $\mu_{2}=1.2 \pm 0.1$ for $T=0.4$ and the distribution function $n m(n)$ versus $n$ obeys the Aboav-Weaire law as shown in Fig. 2. In Fig. 4, the simulated two-cell correlation functions are plotted together with the experimental results for $c f$. Anthurium samples, which have $\mu_{2}=1.1$ [19] showing a reasonable agreement. Two-cell correlation functions for the other two samples, $A$. arborescens and $A$. attenuata, that are shown in Fig. 3, have not been superposed to simula-

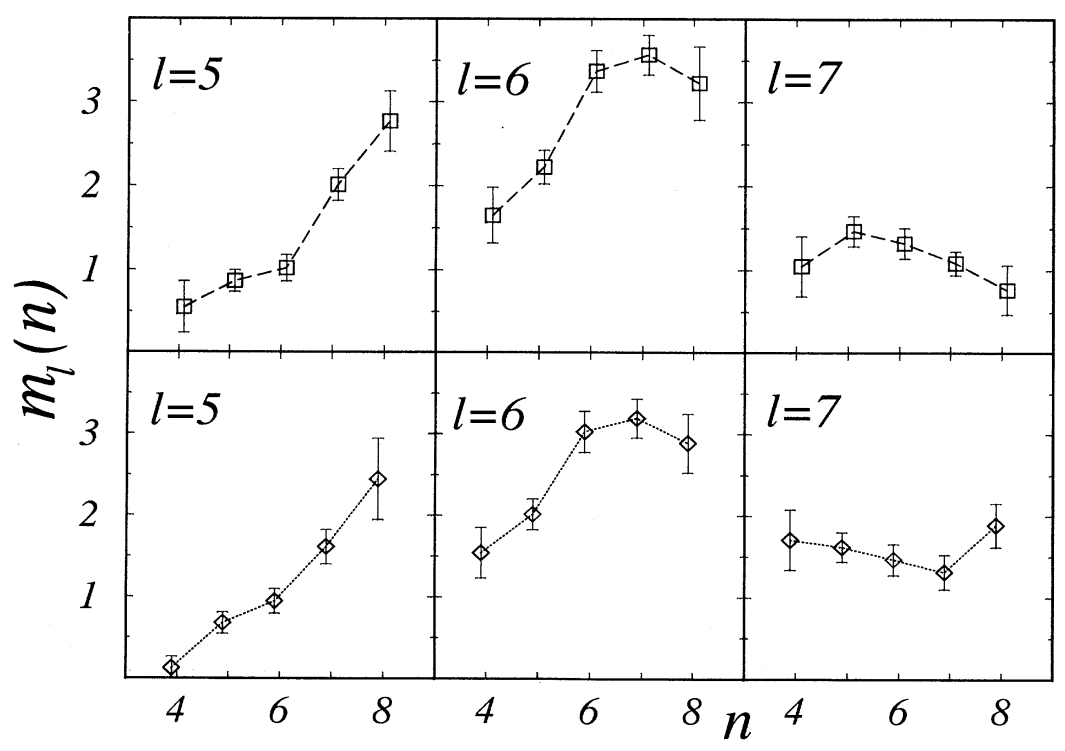

FIG. 3. Two-cell correlation functions $m_{l}(n)$, the average number of $l$-sided cells neighboring $n$-sided ones. Data obtained experimentally for $A$. attenuata (diamonds) and $A$. arborescens (squares). The error bars stand for one standard deviation of the average. 


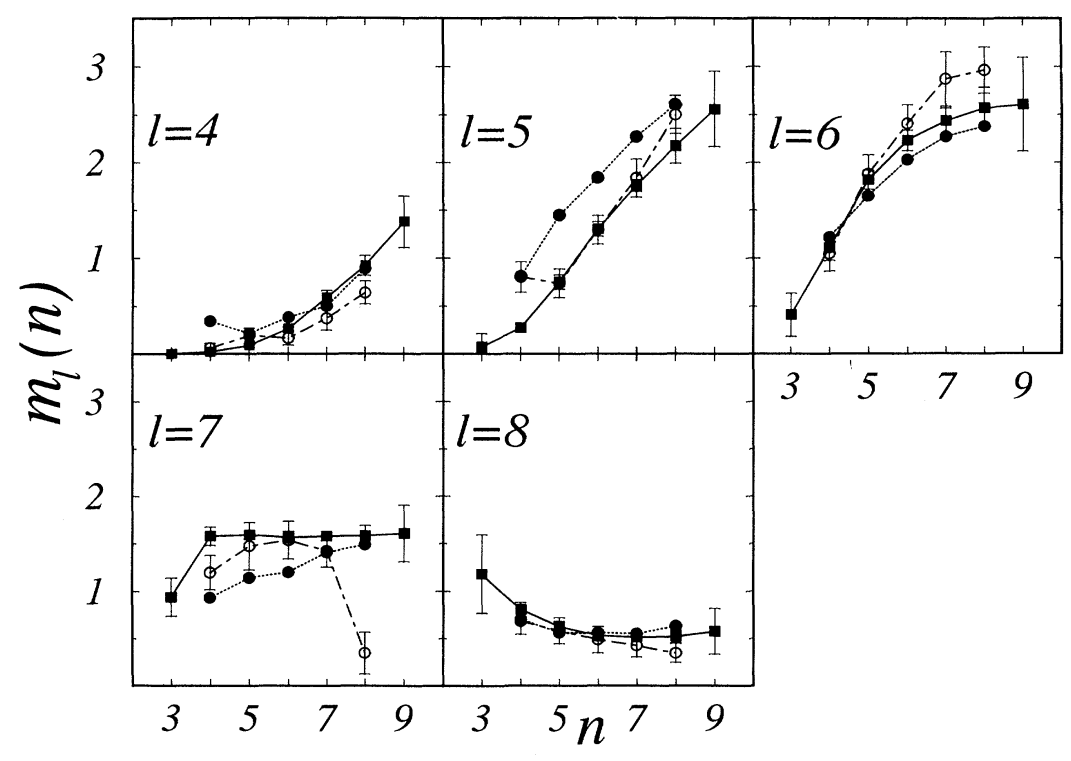

FIG. 4. Numerical simulation results (squares) of two-cell correlation functions $m_{l}(n)$, the average number of $l$-sided cells neighboring $n$-sided ones. For comparison, we plotted also experimental results for $c f$. Anthurium (open circles) and soap froth (full circles).

tion data because they present very different values for the second moment $\mu_{2}, 0.7$, and 0.9 , respectively. The difference between the values of $\mu_{2}$ of different biological samples should be attributed to specific details of the cell growth, as previously discussed [19]. On the other hand, one can obtain a lower value of $\mu_{2}$ in numerical results if the simulation is performed on a triangular lattice where pinning effects are less important.

However, in the absence of a specific mechanism to describe anisotropy, besides the general ones-optimization of the (area)/(perimeter) ratio and minimization of the total perimeter-biological tissues and simulation results reproduce the cellular patterns of soap bubbles or polycrystalline grains, with an important difference: constant average cell area. Also, all the structures, experimental or simulated, clearly verify the Aboav-Weaire law with a slope very near 5 and some of the two-cell correlation functions $m_{l}(n)$ are clearly nonlinear with $n$.

In Ref. [21] it has been argued that the Aboav-Weaire law is a consequence of linear correlation functions, $m_{l}(n)=A_{l}+B_{l} n$, which obviously yield a linear relation between $n m(n)$ and $n$ for all $l$, and that maximize further the entropy, by reducing the number of constraints. These results are based on purely topological arguments, where the area and perimeter of the cells are not considered. A topological simulation confirmed their results.

On the other hand, the simulation and experimental data we presented above show that the Aboav-Weaire law is obeyed by structures that do not always present linear two-cell correlations. The main difference between most of the real cellular systems and topological models lies in the explicit consideration of area, perimeter, and number of sides. These three variables cannot be trivially decoupled because they must fulfill a nonholonomic constraint: there is a maximum possible area for a cell with given perimeter and number of sides [3]. Thus, it seems that diverse real cellular systems may correspond to a single topological structure. Also, cellular patterns are very similar in spite of the differences they may present, but all of them seem to have suffered some growth process where topological constraints compete with some cost function, whose minimization results in decreasing the interfaces or increasing average cell area. There is then a strong interdependence between the number of sides, perimeter, and area. As stated before, this cost function is the surface energy for polycrystalline grains or soap bubbles, but it could be another dynamical mechanism for biological tissues. Purely topological models do not incorporate this competition between topology, geometry, and growth dynamics, and seems not to be suitable to describe natural cellular systems. Further evidence is given in Ref. [3]: a constraint preventing zero-area cells is required to avoid the maximum-entropy solution of many (infinite) zeroarea cells. Topological models do not prevent zero-area cells.

Summarizing, we presented the two-cell correlation functions of biological systems obtained experimentally and through numerical simulations. The simulation results show reasonable agreement with isotropic experimental data and all structures, isotropic or not, obey the Aboav-Weaire law with slopes very near 5 . Two-cell correlation functions are not linear, neither in real systems, nor in simulated ones, where the interface minimization have been explicitly considered, indicating that topology alone is not sufficient to describe natural cellular networks. Therefore, both the universal experimental slope 5 and the linear dependence of $n m(n)$ with $n$ remain open problems.

\section{ACKNOWLEDGMENTS}

We thank Professor M. A. Z. Vasconcellos for useful discussions and A. M. C. D. Moreira for her help in the experimental part of this work. This work has been partially supported by Brazilian agencies $\mathrm{CNPq}$ (Conselho Nacional de Desenvolvimento Científico e Tecnológico), FAPERGS (Fundação de Amparo à Pesquisa do Estado do Rio Grande do Sul), and FINEP (Financiadora de Estudos e Projetos). 
[1] H.V. Atkinsons, Acta Metall. 36, 469 (1988).

[2] D. Weaire and N. Rivier, Contemp. Phys. 25, 49 (1984).

[3] J.R. Iglesias and R.M.C. de Almeida, Phys. Rev. A 43, 2763 (1991).

[4] F.T. Lewis, Anat. Rec. 38, 341 (1928).

[5] R. Delannay, G. Le Caër, and M. Khatun, J. Phys. A 25, 6193 (1992).

[6] H. Flyvbjerg and C. Jeppesen, Phys. Scr. T38, 49 (1991).

[7] J. Stavans, E. Domany, and D. Mukamel, Europhys. Lett. 15, 479 (1991).

[8] J. A. Glazier, M. P. Anderson, and G. Grest, Philos. Mag. B 62, 615 (1990).

[9] M.P. Anderson, D.J. Srolovitz, G.S. Grest, and P.S. Sahni, Acta Metall. 32, 783 (1984).

[10] D.J. Srolovitz, M.P. Anderson, P.S. Sahni, and G.S. Grest, Acta Metall. 32, 793 (1984).

[11] D. Weaire and J.P. Kermode, Philos. Mag. B 48, 245 (1983).

[12] D. Weaire and J.P. Kermode, Philos. Mag. B 50, 379
(1984).

[13] J.Wejchert, D. Weaire, and J.P. Kermode, Philos. Mag. B 53, 15 (1986).

[14] V.E. Fradkov, L.S. Schvindlerman, and D.G. Udler, Philos. Mag. B 55, 289 (1987).

[15] J.C.M. Mombach, R.M.C. de Almeida, and J.R. Iglesias, Phys. Rev. E (to be published).

[16] D. A. Aboav, Metallography 13, 43 (1980).

[17] D. A. Aboav, Metallography 16, 265 (1983).

[18] D. A. Aboav, Metallography 17, 383 (1984).

[19] J.C.M. Mombach, M.A.Z. Vasconcellos, and R.M.C. de Almeida, J. Phys. D 23, 600 (1990).

[20] J. A. Glazier, Ph.D. thesis, The University of Chicago, Chicago, 1989.

[21] M.A. Peshkin, K.J. Strandburg, and N. Rivier, Phys. Rev. Lett. 67, 1803 (1991).

[22] A. M. Kraynik, Annu. Rev. Fluid Mech. 20, 325 (1988).

[23] F. Graner and J.A. Glazier, Phys. Rev. Lett. 69, 2013 (1992). 


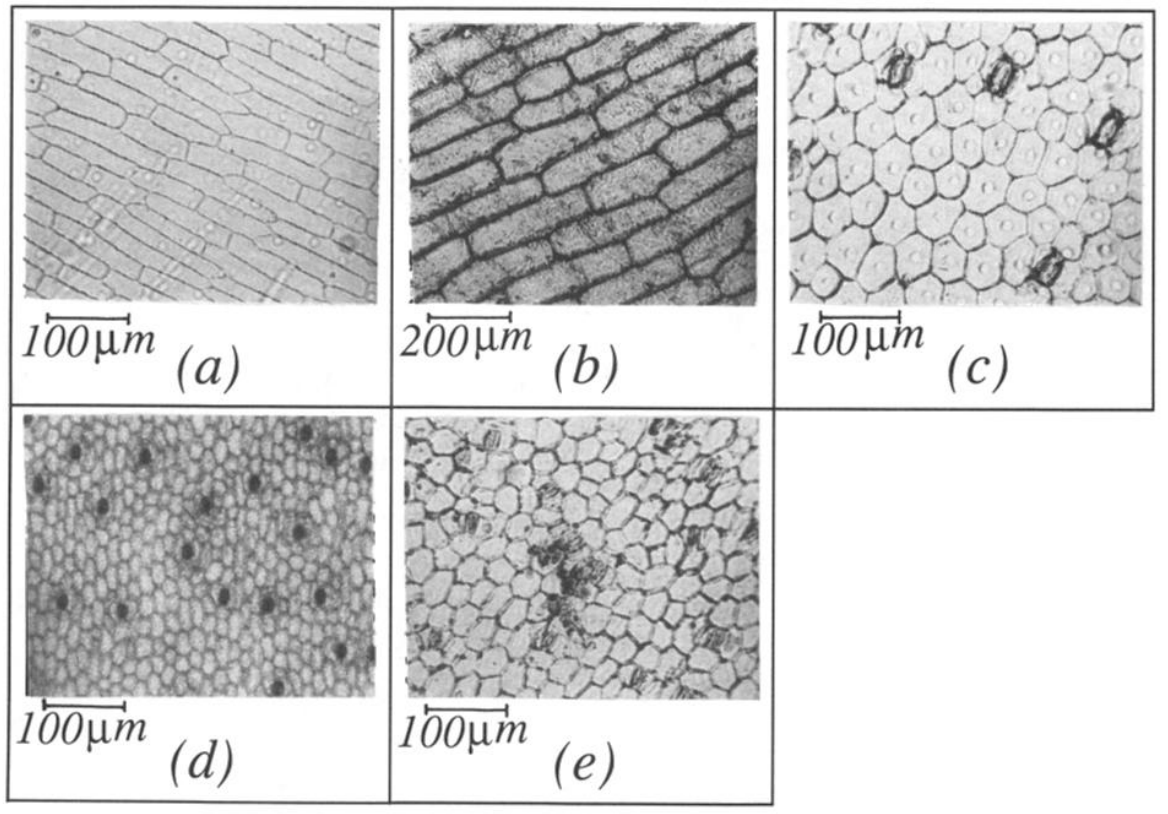

FIG. 1. Representative images of the epidermal tissue [19] for (a) A. sativum, (b) $A$. cepa, (c) A. attenuata, (d) $A$. arborescens, and (e) $c f$. Anthurium. 